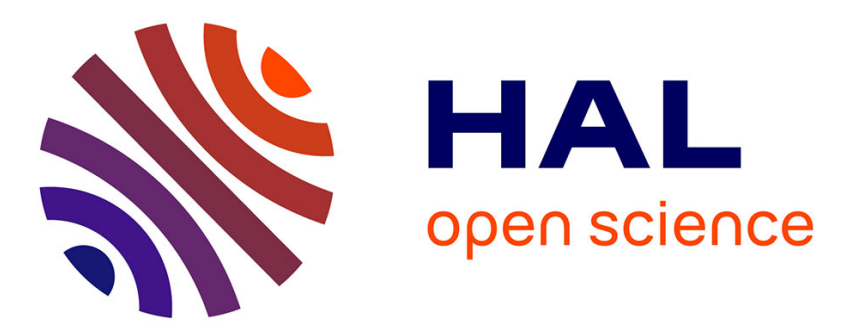

\title{
Surface Control of Doping in Self-Doped Nanocrystals
}

Adrien Robin, Clément Livache, Sandrine Ithurria, Emmanuelle Lacaze, Benoit Dubertret, Emmanuel Lhuillier

\section{To cite this version:}

Adrien Robin, Clément Livache, Sandrine Ithurria, Emmanuelle Lacaze, Benoit Dubertret, et al.. Surface Control of Doping in Self-Doped Nanocrystals. ACS Applied Materials \& Interfaces, 2016, 8 (40), pp.27122 - 27128. 10.1021/acsami.6b09530 . hal-01438644

\section{HAL Id: hal-01438644 https://hal.science/hal-01438644}

Submitted on 2 Jul 2020

HAL is a multi-disciplinary open access archive for the deposit and dissemination of scientific research documents, whether they are published or not. The documents may come from teaching and research institutions in France or abroad, or from public or private research centers.
L'archive ouverte pluridisciplinaire HAL, est destinée au dépôt et à la diffusion de documents scientifiques de niveau recherche, publiés ou non, émanant des établissements d'enseignement et de recherche français ou étrangers, des laboratoires publics ou privés. 


\title{
Surface Control of Doping in self-doped Nanocrystals
}

\author{
Adrien Robin ${ }^{1,2}$, Clément Livache ${ }^{1,3}$, Sandrine Ithurria ${ }^{1}$, Emmanuelle Lacaze $^{3}$, Benoit Dubertret ${ }^{1 *}$, \\ Emmanuel Lhuillier ${ }^{3 *}$ \\ ${ }^{1}$ Laboratoire de Physique et d'Etude des Matériaux, ESPCI-ParisTech, PSL Research University, \\ Sorbonne Université UPMC Univ Paris 06, CNRS, 10 rue Vauquelin 75005 Paris, France. \\ ${ }^{2}$ Nexdot, Biocitech, 102 avenue Gaston Roussel, 93230 Romainville, France \\ ${ }^{3}$ Sorbonne Universités, UPMC Univ Paris 06, CNRS-UMR 7588, Institut des NanoSciences de Paris, 4 place \\ jussieu, 75005 Paris, France
}

\begin{abstract}
Self-doped nanocrystals raise great interest for infrared (IR) optoelectronics because their optical properties span from near IR to far IR. However, their integration for photodetection requires a fine understanding of the origin of their doping and also a way to control the magnitude of the doping. In this paper, we demonstrate that a fine control of the doping level between 0.1 and 2 electrons per dot is obtained through ligand exchange. The latter not only affects the inter-particle coupling but also their optical properties because of the band-shift resulting from the presence of surface dipoles. We demonstrate that self-doping is a bulk process and that surface dipoles can control its magnitude. We additionally propose a model to quantify the dipole involved with each ligand. We eventually use the ligand design rule previously evidenced to build a near infrared photodetector on a soft and transparent substrate. The latter significantly improves the performance compared to previously reported Colloidal Quantum Dots (CQD) based photodetectors on plastic substrate operated in the telecom wavelength range.
\end{abstract}

Keywords: HgSe, self-doping, Colloidal quantum dot, electrolyte gating, photoresponse, infrared.

*To whom correspondence should be addressed. E-mail: el@insp.upmc.fr and benoit.dubertret@espci.fr 


\section{Introduction}

While nanocrystals are attracting a large interest for their tunable optical properties from UV to $\mathrm{THz}^{1}$, the control of their electronic properties remains more challenging, thus slowing down their integration into optoelectronic devices ${ }^{2}$. The open question of the control of electrical doping is as of today of utmost interest. Indeed, in spite of large progresses over the recent years ${ }^{3,4,5}$, no systematic strategies have been proposed to tune the electrical doping ${ }^{6}, 7$ of colloidal quantum dots (CQD). Partial cation exchange ${ }^{4,8}$ is one of the most promising path to achieve the bulk doping of CQD. However, even in the low doping regime of 1 dopant per CQD, this actually corresponds to a large doping regime for bulk semiconductors $\left(>10^{18}\right.$ $\mathrm{cm}^{-3}$ ). Complementary methods are then needed to achieve a fine control of the carrier density in a range of 0.1 to 1 carrier per nanocrystal.

This question is especially interesting to finely tune the doping level of self-doped nanocrystals (i.e. doping is obtained without process to induce it). Recently, it has been shown that mercury chalcogenides nanocrystals of $\mathrm{HgS}^{9,10}$ and $\mathrm{HgSe}^{11}$ are naturally $n$-doped under ambient conditions ${ }^{1}$. They typically present 1 to 2 carriers per nanocrystal and consequently exhibit interband and intraband absorption ${ }^{1}$ in the mid or far IR. Thanks to this long wavelength absorption, HgSe CQD have been successfully integrated as mid and far infrared photodetectors ${ }^{1,11}$. Nevertheless, the photocurrent modulation remains poor compared to the dark current, and the specific detectivity value (i.e. signal-to-noise ratio) remains smaller than the values reported for photodetectors based on epitaxial grown narrow band gap semiconductors (HgCdTe, InSb, type II superlattice...). Reducing the dark current is then mandatory to improve the photodetection performances. To fulfill this objective, controlling the doping level and the resulting carrier density is of utmost interest. Nevertheless, very little is known on the origin of this doping and on its control.

In this paper, we first describe some of the current limitations of devices based on these self-doped nanocrystals. In an effort to provide a better understanding of the doping, we evidence that the doping level can be finely tuned thanks to a change of surface chemistry. We propose a model where we ascribe the change in doping density to the magnitude of the surface dipoles originating from the ligands. This model is then used to quantify the value of the surface dipole directly from optical measurements. The resulting simple surface chemistry rules are then applied to design a near IR photodetector on soft and transparent electrodes.

\section{Discussion}

HgSe nanocrystals combine (i) a narrow band gap originating from the semi-metal character of the bulk material with (ii) a self-doping which leads to optical features in the infrared. For $5 \mathrm{~nm}$ CQD (see Figure $1 \mathrm{a})$, the interband band edge is typically around $6000 \mathrm{~cm}^{-1}(0.8 \mathrm{eV})$. This ground state transition, from the 1s state of the valence band to the $1 \mathrm{~s}$ state of the conduction band, is almost fully bleached. Since the nanocrystals are capped with dodecanethiol (DDT) ligands at the end of the synthesis, only the transition from the $1 \mathrm{~s}$ state of the valence band to the $1 \mathrm{p}$ state of the conduction band appears at higher energy ${ }^{12}$ $\left(\sigma \approx 8500 \mathrm{~cm}^{-1}\right)$, Figure $1 \mathrm{~b}$. This transition is better revealed after ligand exchange, see Figure 2 . In addition, the CQD present a second optical feature, at $2500 \mathrm{~cm}^{-1}(310 \mathrm{meV})$, relative to a transition within the conduction bands ${ }^{9,11}$, see Figure $1 \mathrm{~b}$. The latter is an immediate proof of their doped character. 
To design a photodetector from this material, a fine control of the doping is mandatory. Indeed, for midinfrared photodetectors, only the intraband contribution of the absorption is useful and an accurate doping value of two electrons per dot is optimal to combine the intraband absorption and prevents dark current through the 1s state. Indeed, a value of 2 carriers per dot fully fills the 1s states: the Pauli blockade thus prevents transport between the $1 \mathrm{~s}$ states. In this case, the transport only occurs via the $1 \mathrm{p}$ states, which requests a thermal or photon activation. On the other hand, for a near infrared photodetector, the intraband transition energy is too low and generates more dark current. A lower doping value is thus more appropriated. In this sense, a careful tuning of the doping level is required.
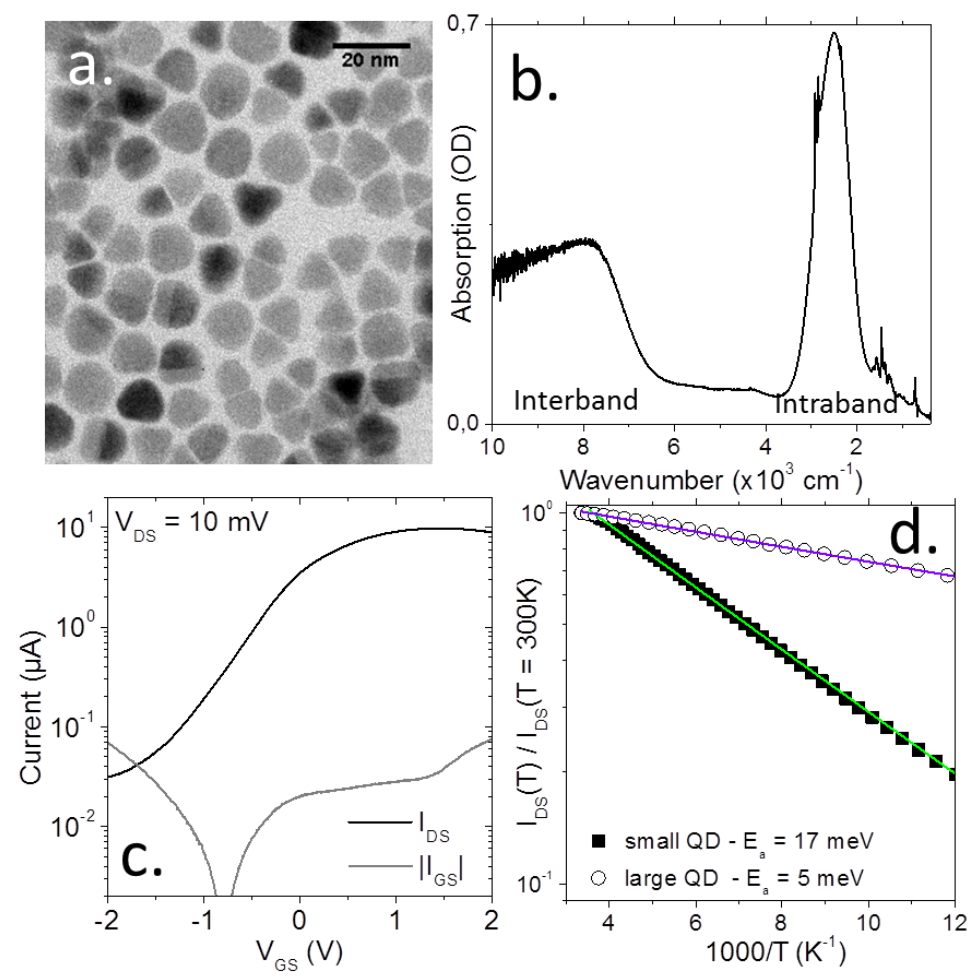

Figure 1 a. TEM image of HgSe colloidal quantum dots. b. Infrared spectrum of the same HgSe CQD. c. Transfer curve (drain and gate current as a function of gate voltage) for an electrolytic transistor which channel is made from HgSe CQD capped with sulfide ligands. The drain bias is set to $10 \mathrm{mV}$. The gate bias step is $1 \mathrm{mV}$. d. Current as a function of temperature for a thin film made from HgSe CQD. The experimental points are round (large CQD $>10 \mathrm{~nm}$ ) and square (small CQD of $5 \mathrm{~nm}$ ). The solid lines are Arrhenius fits of the experimental data.

The doping of the HgSe CQD is also confirmed by transport measurements conducted in an electrolytic transistor configuration. The transfer curve of such transistor is presented in Figure 1c and presents an $n$ type character and a current modulation of roughly two orders of magnitude. Under illumination, the current modulation remains limited $(\approx 100 \%)$. In narrow band gap materials, the large number of thermally activated carriers generates a high dark current which usually prevails over the photocurrent modulation $^{13}$. The most common strategy to reduce this dark current is to cool the device. We consequently conducted transport measurements at low temperature and observed an Arrhenius dependence of the current, ${ }^{14}$ with a low value of the activation energy, see Figure $1 \mathrm{~d}$. The latter ranges 
from 5 to $20 \mathrm{meV}$, depending on the size of the CQD. Indeed, the larger the nanocrystal is, the lower is the activation energy. These activation energies are one order of magnitude smaller than the optical intraband transition energy $(\approx 300 \mathrm{meV}$ for the small CQD). As a result, only a small improvement of the signal-todark ratio is achieved by cooling this material.

Such behavior immediately raises the question of the driving parameter of the activation energy. Since the latter is related to the doping magnitude ${ }^{13,15}$, the origin of the self-doping and the strategies to tune its magnitude need to be evidenced. Mercury chalcogenides are known to present electronic properties sensitive to ambient condition after ligand exchange ${ }^{16}$, necessary to increase the inter-quantum dot coupling and in turn achieve an electrical conductivity. We thus investigated the effect of ligands in HgSe films on the electrical and optical properties. We conduct solid state ligand exchange from dodecanethiol to short organic ligands (ethanedithiol) or ionic ligands $\left(\mathrm{SCN}^{-}, \mathrm{I}^{-}, \mathrm{S}^{2-}, \mathrm{As}_{2} \mathrm{~S}_{3}\right)$. We observe that not only the conductance of the film switches from an insulating behavior $(R>100 \mathrm{M} \Omega)$ to a conductive state $(R>1-10$ $k \Omega$ ), but also the absorbance spectrum is affected, see Figure $2 a$ and $b$. The relative interband contribution of the signal is indeed increased compared to the intraband contribution, which means that the doping level is reduced. This trend is observed for all sizes of CQD but is particularly striking for the smallest nanoparticles, see Figure $2 c$.
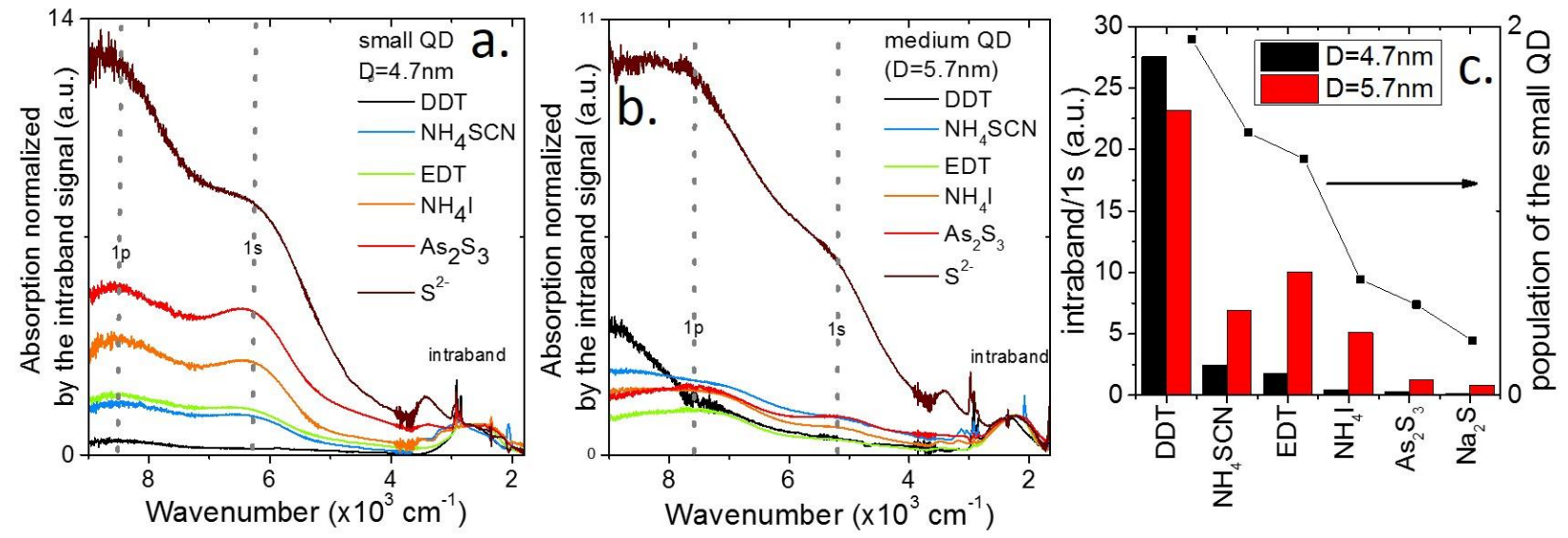

Figure 2. Absorption spectra of a. small sized $(4.7 \mathrm{~nm})$ and b. medium sized $(5.7 \mathrm{~nm}) \mathrm{HgSe}$ nanocrystals capped with different ligands. c. Plot of the ratio of the intraband signal divided by the fitted magnitude of $1 \mathrm{~s}$ interband signal for two sizes of nanocrystals. The related population of the CQD is given on the right scale for the small sized CQD (4.7 $\mathrm{nm}$ diameter).

We can relate the relative absorption of the interband and intraband signal to the population of the $1 \mathrm{~s}$ state of the conduction band. Assuming a density of state of two electrons on the $1 \mathrm{~s}$ states ${ }^{17}$ due to spin degeneracy, the interband absorption, $\alpha_{\text {inter }}$ relates to the population of this level, $n(1 s)^{18}$, though the relation $\alpha_{\text {inter }} \propto 2-n(1 s)$. Similarly, the intraband absorption is simply proportional to this population $\alpha_{\text {int ra }} \propto n(1 s)$, as long as the $1 p$ states is empty, which is the case for the smallest CQD ${ }^{10}$. We can thus estimate the population of the state by $n(1 s)=\frac{2}{1+\frac{\alpha_{\text {int } e r}}{\alpha_{\text {int } r a}}}$, see Figure $2 c$. We typically observe a drop of a factor 10 of the population of the 1 s state while we switch from the initial long DDT ligands $(n(1 s) \approx 2)$ 
to ligands such as $\mathrm{As}_{2} \mathrm{~S}_{3}$ or $\mathrm{S}^{2-}$ ( 0.2 electron per dot), see Figure $2 \mathrm{c}$. Recently, it was also pointed by Deng et $a l^{19}$ that the growth of $\mathrm{CdS}$ shell on a HgSe core can strongly affect the doping level up to the point where the intraband transition is no longer observed, see figure S1.

These observations bring us to the question of how the presence of a self-assembled monolayer (i.e. ligands) can affect the electronic properties of a surface. While this question has been intensively discussed for bulk materials ${ }^{20}$, the impact of surface dipole on confined nanoparticles remains far less discussed ${ }^{9}$. For nanoparticles, it has been evidenced using STM ${ }^{21}$ and X-ray photoemission spectroscopy ${ }^{22}{ }^{23}$ that the band energies are affected by the choice of the capping ligands. Indeed, the magnitude of the related dipole can tune the absolute energy position of the gap over almost $1 \mathrm{eV}$ in the case of $\mathrm{PbS}^{22}$. Such a shift could also explain the large ligand induced tuning of the doping level for self-doped mercury chalcogenides nanocrystals.

The band diagram of HgSe CQD is given in Figure 3a. Two characteristic energies are present: $(i)$ the interband band edge (BE) energy and the (ii) intraband energy. In presence of ligands, the pristine band diagram of the CQD gets affected by the electric field resulting from the ligand induced dipoles. The electrostatic response of the CQD to a dipole pointing its negative charge toward its surface is to bring positive charges to the surface, which in turn bend the band, see Figure $3 \mathrm{~b}$. While the Fermi level of the ligands and of the nanocrystal equilibrates, a shift of the vacuum level occurs. This induces a similar shift of the band with respect to the Fermi level. In other words, a ligands decorated QD presents a shifted band diagram compared to the pristine CQD. The shift of the band is responsible for a change of the electronic population level within the CQD.
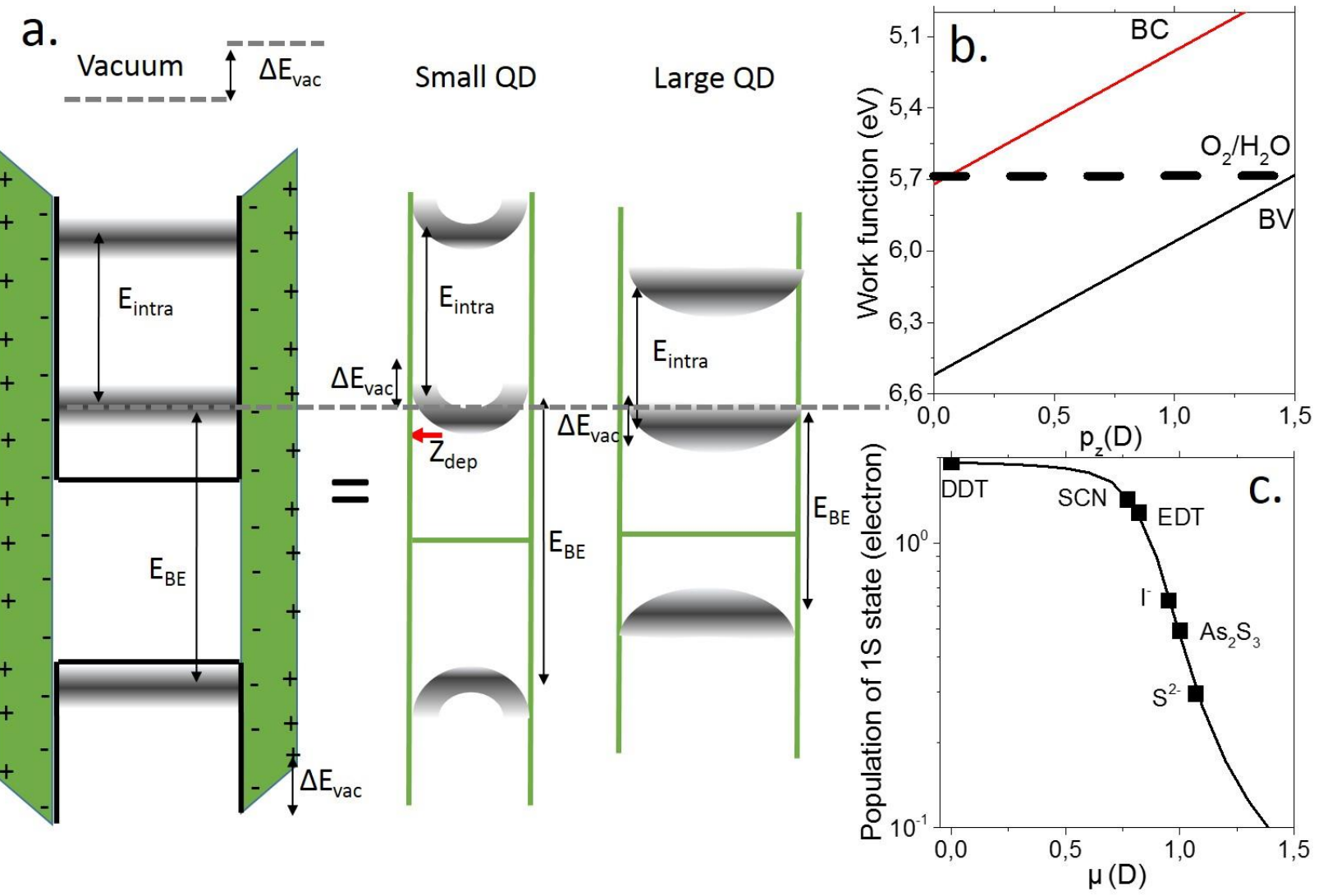
Figure 3 a. Band diagram of a self-doped nanocrystal in absence of surface dipole (left) and once surface dipoles are added (central and right part). The addition of dipoles on the nanocrystal surface leads to an electric field which shifts the vacuum level and the relative position of the band gap with respect to the Fermi level (grey dotted line). b. Plot of the conduction and valence band energy versus vacuum as a function of the surface dipole. c. Plot of the 1 s state population as a function of the intensity of the surface dipole.

One can estimate the shift of the band thanks to the relation $20,22,24$ $\Delta E_{v a c}=-N\left[\frac{\mu_{\perp}(\text { ligand })}{\varepsilon_{0} \varepsilon_{\text {ligand }}}+\frac{\mu_{H g \text {-ligand }}}{\varepsilon_{0} \varepsilon_{H g X}}\right]$ where $N$ is the surface density of dipole, $\mu_{\perp}$ (ligand) is the component of the intrinsic ligand dipole normal to the surface, $\varepsilon_{0}$ is the vacuum permittivity, $\varepsilon_{\text {ligand }}$ the dielectric constant of the ligand, $\mu_{H g \text {-ligand }}$ the dipole relative to the interface between the crystal surface and the ligand and $\varepsilon_{H g X}$ the dielectric constant of the nanocrystal. For the sake of simplicity, we assume that this surface band shift is translated to a band shift in volume $\Delta E_{v a c}=-N \frac{\mu_{\perp}(\text { ligand })}{\varepsilon_{0} \varepsilon_{\text {ligand }}}$, where $\mu_{\perp}($ ligand $)$ is the averaged dipole magnitude over the whole nanocrystal volume.

Bulk mercury chalcogenides present large work function values ${ }^{25}$ (around $6 \mathrm{eV}$ for II-VI compounds), which, together with their narrow band gap nature, implies that their conduction band can be lower than the $\mathrm{O}_{2} / \mathrm{H}_{2} \mathrm{O}$ redox couple potential $\left(-1.23 \mathrm{~V} \mathrm{H}^{+} / \mathrm{H}_{2}\right.$ or $5.7 \mathrm{eV}$ below vacuum level), see Figure $3 \mathrm{~b}$. This actually explains why $\mathrm{HgS}$ and $\mathrm{HgSe} \mathrm{CQD}$ can be stable under a negatively charged form and why HgTe, whose band structure is shifted toward the vacuum level, is not. In those large work function conditions, the water is a reducing agent for the $\mathrm{QD}$ according to the following redox equation $2 \mathrm{QD}+\mathrm{H}_{2} \mathrm{O} \longrightarrow 2 \mathrm{QD}^{-}+1 / 2 \mathrm{O}_{2}+2 \mathrm{H}^{+}$. This hypothesis is further confirmed by the fact that conducting the ligand exchange in presence of water is not affecting the absorption spectrum, see Figure S2. This model also explains why the smallest HgSe CQD (3 $\mathrm{nm}$ and less) do not present intraband absorption. Indeed, they are so confined that their conduction band remains above the redox potential of the $\mathrm{O}_{2} / \mathrm{H}_{2} \mathrm{O}$ couple. Similarly, when dipoles are added on the CQD surface, the conduction band of the CQD shifts above the $\mathrm{O}_{2} / \mathrm{H}_{2} \mathrm{O}$ redox couple potential and the previous reduction can no longer occur. The stable form of the CQD becomes the neutral form, see Figure $3 \mathrm{~b}$. This allows us to answer a key question regarding the doping origin, which is to know if it rather comes from bulk or surface effect. Our experimental data pledges for a bulk doping, since that the larger the CQD, the smaller is change of the optical signature (ratio of intraband on interband) and in turn the doping is affected by a change of the surface dipole, see Figure 2c.

In the framework of this model, we can quantify the change of carrier density within the 1s state of the conduction band as a function of the surface dipole magnitude. We assume that the dipole density is 3 $\mathrm{nm}^{-2}$, which corresponds to a full coverage of the surface (1 dipole per cation), consistent with experimental values ${ }^{26}$ reported for chalcogenides nanocrystals. This value is actually an effective value and is averaged over the different exposed facets of the CQD, which present some variations of the capping density. 
According to the optical absorption, in presence of DDT ligands the first interband transition is fully bleached which pledges for a Fermi level at the top of the $1 \mathrm{~s}$ state. This corresponds to 2 electrons per nanocrystal.

The carrier density can be calculated by $n_{1 s}=2 \int D O S(E) \times f_{F D}\left(E-E_{F}\right) d E$ where the factor 2 results from the spin degeneracy, the density of state is given by $\operatorname{DOS}(E)=\frac{\Gamma}{2 \pi} \frac{1}{\left(\frac{\Gamma}{2}\right)^{2}+\left(E-E_{1 S}\right)^{2}}$ where gamma is the energy level lorentzian broadening and is fitted with the experimental spectrum given in Figure $2 \mathrm{a}\left(\Gamma=800 \mathrm{~cm}^{-1} \approx 100 \mathrm{meV}\right)$ and finally the Fermi Dirac population factor is given by $f_{F D}\left(E-E_{F}\right)=\frac{1}{1+\exp \left(\frac{E-E_{F}}{k_{b} T}\right)}$. Taking $E^{\prime}=E-E_{F} \quad$ and considering the difference $\Delta E_{V a c}=\Delta\left(E_{1 S}-E_{F}\right)$, we can $\quad$ write $n_{1 s}=2 \int \frac{\Gamma}{2 \pi} \frac{1}{\left(\frac{\Gamma}{2}\right)^{2}+\left(E^{\prime}-\Delta E_{\text {Vac }}\right)^{2}} \times \frac{1}{1+\exp \left(\frac{E^{\prime}}{k_{b} T}\right)} d E^{\prime \prime}$. Considering that $\Delta E_{V a c}=\Delta\left(E_{1 S}-E_{F}\right) \approx-N\left[\frac{\mu_{\perp}(\text { ligand })}{\varepsilon_{0} \varepsilon_{\text {ligand }}}\right]$ and taking $\varepsilon_{\text {ligand }}=2,{ }^{17}$ we can plot the population of the $1 \mathrm{~s}$ state $n_{1 s}$ as a function of the dipole magnitude $\mu_{\perp}$ (ligand) on the Figure 3c. According to the population of the $1 \mathrm{~s}$ state that we have measured thanks to optical spectroscopy (Figure $2 \mathrm{c}$ ), we can now estimate the change of the dipole value for each ligand. In other word, this model allows us to estimate the surface dipole related to a ligand using only optical spectroscopy. For the short ligands, this value ranges from 0.7 to $1.1 \mathrm{D}$, which is consistent with the typical value expected from DFT simulations ${ }^{27,28}$. In the case of $\mathrm{S}^{2-}$, we can assimilate the dipole length to the Hg-S bond length $(2.53 \AA)$ and estimate the gating charge as 0.08 electrons per dipole.

We finally take advantage of this surface tunability of the doping to design a near IR photodetector on a soft and transparent substrate. Since we previously demonstrated that $S^{2-}$ ligands lead to a full disappearing of the intraband transition ${ }^{9}$, we use this strategy as a way to reduce the dark current in the $\mathrm{HgSe}$ based photodetector while the sample is illuminated by a $1.55 \mu \mathrm{m}$ laser source.

As organic electronics, CQD based devices offer the significant advantage of being processable on a large range of substrates without the constrain of an epitaxial growth. However, organic materials can hardly access the telecom wavelength range. Furthermore, very little work has been dedicated to the integration of narrow band gap CQD on such plastic substrates. The Kagan's group ${ }^{29}$ has demonstrated some complementary electronic building block from PbSe CQD, but they did not report their use as photodetector. The Kim's group also pioneered the design of such devices with the use of HgTe CQD ${ }^{30,31}$. Nevertheless, the achieved level of performances remains quite limited, with photoresponsivity of the order of few $\mathrm{nA} . \mathrm{W}^{-1}$ for illumination at a telecom wavelength ${ }^{32,33,34}$. 

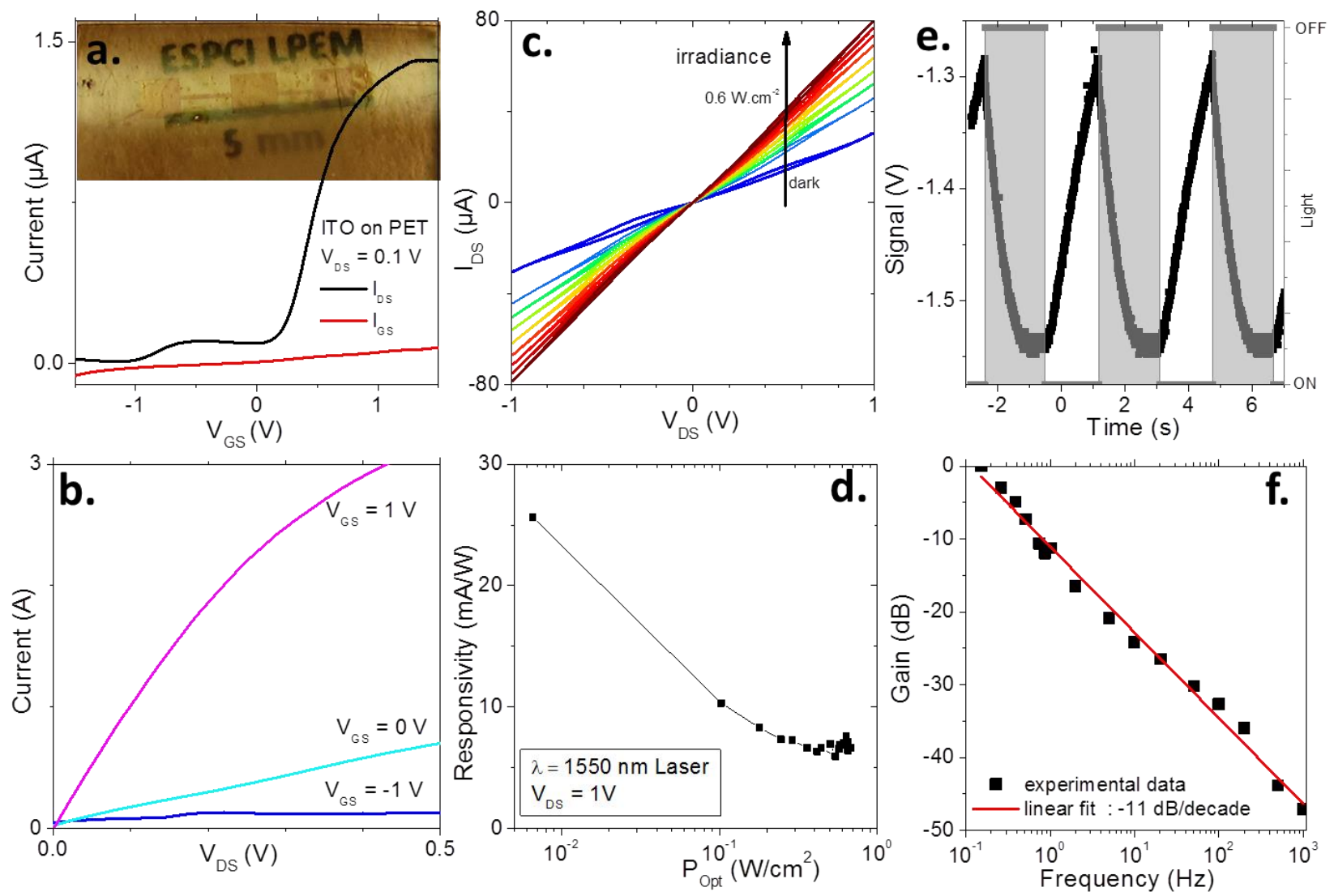

Figure $4 a$. Transfer curve and $b$. IV curves under different gate bias of an ion-gel transistor made from a film of HgSe CQD capped with $\mathrm{S}^{2-}$ and deposited on ITO electrodes on a PET substrate. The inset of $a$. is a picture of the film and the electrodes. c. Photoresponse under dark conditions and under illumination by $a$ $1.55 \mu \mathrm{m}$ laser at different laser intensities, $d$. related power dependence of the photoresponsivity, e. change of the current signal under light illumination with a $808 \mathrm{~nm}$ laser as a function of time and $f$. dependence of the photocurrent versus illumination modulation frequency of a film of HgSe CDQ capped with $\mathrm{S}^{2-}$ on ITO electrodes on a PET substrate.

We designed ITO electrodes on a PET substrate, both of them being transparent in the telecom wavelength range, see figure S3. A film of $\mathrm{HgSe}$ CQD capped with $\mathrm{S}^{2-}$ ligand has been prepared on these electrodes. The film then presents only the interband absorption with an excitonic structure perfectly optimized for $1.55 \mu \mathrm{m}\left(6450 \mathrm{~cm}^{-1}\right.$, see Figure 2a) signal. In a transistor configuration, we observe a large current modulation which can go above two orders of magnitude, see Figure 4a-b. The material remains $n$-type. Under illumination, the device presents a positive photoresponse and the current modulation can be as large as a factor 2.5 , see Figure 4c. The related photoresponsivity is in the $10-20 \mathrm{mAW}^{-1}$ range, see Figure $4 \mathrm{~d}$, which is far larger than the previously reported values for CQD based photodetectors on plastic substrates operating at the same wavelength. However, one key current limitation remains their slow response ( $>1 \mathrm{~s}$, see Figure $4 \mathrm{e}$ and $\mathrm{S4}$ ), which promptly drops while the signal frequency increases, see Figure $4 f$. 
Future works are needed to find strategies to speed up this response time at least in the $50 \mathrm{~ms}$ range to be compatible with video frame rates. The design of shorter devices with a smaller inter-electrodes spacing reducing the transit time will certainly be helpful.

\section{Conclusion}

We elucidate some questions regarding the origin of the self-doping in mercury chalcogenides CQD. We propose that the doping results from the bulk reduction of the CQD by water because of their large work function and the narrow band gap of the $\mathrm{HgS}$ and $\mathrm{HgSe}$ compounds. We further demonstrate that the doping can be tuned in the 0.1 to 1 carrier per dot range thanks to ligand exchange. The related tuning of the surface dipole changes indeed the relative energy of the conduction band with respect to the $\mathrm{O}_{2} / \mathrm{H}_{2} \mathrm{O}$ couple. We propose a quantitative model to determine the magnitude of the dipole related to each ligand and evidence that a change of $1 \mathrm{D}$ of the surface dipole correspond to a decrease of the doping level by a factor 10. Eventually, we use this method to design a near IR photodetector. We choose a ligand which fully suppresses the intraband absorption and optimizes the absorption signal in the telecom wavelength range. Compared with previous reports, the photoresponsivity of our detector is enhanced by almost six orders of magnitude. 


\section{Methods}

\section{Chemicals}

Selenium powder (Sigma-Aldrich, 99.99\%), selenium disulfide ( $\mathrm{SeS}_{2}$, Sigma-Aldrich), mercury acetate $\left(\mathrm{Hg}(\mathrm{OAc})_{2}\right.$, Sigma-Aldrich), trioctylphosphine (TOP, Cytek, 90\%), dodecanethiol (DDT, Sigma-Aldrich), oleic acid (Sigma-Aldrich, 90\%), oleylamine (Acros, 80-90\%), arsenic trisulfide ( $\mathrm{As}_{2} \mathrm{~S}_{3}$,Strem, 99.9\%), propylamine (Sigma-Aldrich, 98\%), n-methylformamide (NMFA, VWR, 98\%), lithium perchlorate $\left(\mathrm{LiClO}_{4}\right.$, Sigma-Aldrich, 98\%), polyethylene glycol 6k (PEG 6k, $\mathrm{M}_{\mathrm{W}}=6 \mathrm{~kg} \cdot \mathrm{mol}^{-1}$, Fluka), ethanol absolute anhydrous (99.9\%, Carlo Erba) acetone (Carlo-Erba, 99.8\%), ammonium iodide ( $\mathrm{NH}_{4} 1$, Sigma-Aldrich, 99\%), ammonium thiocyanate $\left(\mathrm{NH}_{4} \mathrm{SCN}\right.$, Sigma-Aldrich, 99\%), 1,2-ethanedithiol (EDT, Fluka, 98.0\%), sodium sulfide nonahydrate $\left(\mathrm{Na}_{2} \mathrm{~S}, 9 \mathrm{H}_{2} \mathrm{O}\right.$, Sigma-Aldrich $\left.98.0 \%\right)$.

All chemicals are used as received, except oleylamine which is centrifuged before use.

\section{Precursors}

$1.54 \mathrm{~g}$ of Se powder is mixed in $20 \mathrm{~mL}$ of TOP. After sonication, a transparent solution is obtained.

\section{Nanocrystal synthesis}

In a $100 \mathrm{~mL}$ three-neck flask, $1 \mathrm{~g}$ of mercury acetate is mixed with $20 \mathrm{~mL}$ of oleic acid and $50 \mathrm{~mL}$ of oleylamine. The solution is degassed at room temperature, then at $80^{\circ} \mathrm{C}$ for $30 \mathrm{~min}$. The atmosphere is then switched to Ar. At $90^{\circ} \mathrm{C}, 3.2 \mathrm{~mL}$ of TOPSe (AM) is quickly injected and the solution turns dark. After 5 min of reaction, $1 \mathrm{~mL}$ of dodecanthiol is added to quench the reaction while the heating mantle is removed. The content of the flask is split into two $50 \mathrm{~mL}$ Falcons and equivalent volume of ethanol is added to precipitate the solution. After centrifugation, the clear supernatant is discarded and the formed pellet redispersed in fresh toluene. The cleaning procedure is repeated two other times.

\section{Mercury compounds are highly toxic. Handle them with special care!}

\section{Film deposition and solid state ligand exchange procedure}

The cleaned HgSe QDs suspension in toluene is typically diluted between 20 to 100 times in a 9:1 volume ratio hexane:octane mixture. The resulting suspension is light brown and transparent, and has a target optical density at $15000 \mathrm{~cm}^{-1}$ ranging between 0.5 and 1 . Meanwhile, a ligand exchange solution in ethanol is prepared. For $\mathrm{NH}_{4} \mathrm{SCN}$ and $\mathrm{NH}_{4}$ l, a $1 \%$ in weight solution of the compound is prepared; for $\mathrm{Na}_{2} \mathrm{~S}_{2} 9 \mathrm{H}_{2} \mathrm{O}$, a saturated solution is prepared, then filtered at $0.2 \mu \mathrm{m}$ and subsequently de-saturated by doubling the solvent volume; for $\mathrm{As}_{2} \mathrm{~S}_{3}, 1 \mathrm{mg}$ of the latter is partially dissolved in $1 \mathrm{~mL}$ of propylamine, a $1 \%$ in volume of the latter solution in ethanol is then prepared; for EDT, a $1 \%$ solution in ethanol is prepared. A 50 to $100 \mathrm{~nm}$ thick and homogeneous film is then prepared by repeating the following procedure 10 times: a 5 $\mu \mathrm{L}$ droplet of the QDs suspension is drop-casted in air onto the electrodes, then dried in open air. The sample is dipped $1 \mathrm{~min}$ in the ligand exchange solution, then rinsed in pure ethanol for $1 \mathrm{~min}$, then dried in open air. This monolayer-by-monolayer procedure produces a clean crack-free film whose roughness is on the order of the nanocrystal size.

\section{Electrodes on $\mathrm{Si} / \mathrm{SiO}_{2}$ wafer}


The surface of a Si/ $\mathrm{SiO}_{2}$ wafer ( $400 \mathrm{~nm}$ as thickness) is cleaned by sonication in acetone. The wafer is rinsed with isopropanol and finally cleaned using an $\mathrm{O}_{2}$ plasma. $\mathrm{AZ} 5214 \mathrm{E}$ resist is spin-coated and baked at $110^{\circ} \mathrm{C}$ for $90 \mathrm{~s}$. The substrate is exposed under UV though a pattern mask for $2 \mathrm{~s}$. The film is further baked at $125^{\circ} \mathrm{C}$ for $2 \mathrm{~min}$ to invert the resist. Then a $40 \mathrm{~s}$ flood exposure is performed. The resist is developed using a bath of AZ 326 for $32 \mathrm{~s}$, before being rinsed in pure water. We then deposit a $3 \mathrm{~nm}$ chromium layer and a $40 \mathrm{~nm}$ gold layer using a thermal evaporator. The lift-off is performed by dipping the film for in acetone for $1 \mathrm{~h}$. The electrodes are finally rinsed using isopropanol and dried by an air flow. The electrodes are 2 $\mathrm{mm}$ long and spaced by $20 \mu \mathrm{m}$.

\section{Electrodes based on ITO on PET}

Indium tin oxide (ITO) coated on polyethylene terephthalate (PET) $\left(\approx 80 \mathrm{~nm}\right.$ coating with a $60 \Omega / \mathrm{cm}^{2}$ resistance) sheet are purchased from Sigma-Aldrich. The film is rinsed using acetone and then isopropanol before being dried. AZ 5214E resist is spin-coated and then baked for $90 \mathrm{~s}$ on a $110^{\circ} \mathrm{C}$ hot-plate. The film is then exposed to UV for $4 \mathrm{~s}$ though a shadow mask. The resist is then developed for $45 \mathrm{~s}$ in AZ 726 and rinsed in pure water. The naked ITO is then etched using $25 \% \mathrm{HCl}$ solution for $15 \mathrm{~s}$ and then quickly rinsed in pure water. The lift-off of the resist is made by dipping the substrate in acetone for $5 \mathrm{~min}$ and then rinsing the film with isopropanol. The designed electrodes are interdigitated electrodes with $50 \mu \mathrm{m}$ spacing. Each electrode is itself $50 \mu \mathrm{m}$ large and $1 \mathrm{~mm}$ long. The total active area is $1 \mathrm{~mm}^{2}$. A side gate electrode is also present for electrolyte gating.

\section{Electrolyte preparation}

$50 \mathrm{mg}$ of $\mathrm{LiClO}_{4}$ are mixed with $230 \mathrm{mg}$ of PEG on a hot plate in an Ar filled glove box at $170^{\circ} \mathrm{C}$ for $2 \mathrm{~h}$.

\section{Transistor fabrication}

Electrical measurements are conducted in air and made using a Keithley $2634 \mathrm{~b}$ as source-meter. Transistor measurements are conducted by biasing the drain source under low bias (10 or $50 \mathrm{mV}$ ) and applying a varying gate bias. The gate bias step is $1 \mathrm{mV}$ and the sweep rate $2 \mathrm{mV} \cdot \mathrm{s}^{-1}$ for electrolyte gating.

\section{Material characterization}

For TEM imaging, the solution of CQD is drop-casted on a copper TEM grid and degassed overnight under vacuum. The TEM images are then obtained from a JEOL 2010 microscope.

IR optical absorption is measured in a transmission configuration using a Bruker Vertex 70 spectrometer. A global source equivalent to a $700^{\circ} \mathrm{C}$ blackbody is used as source and DTGS detector. Spectra are typically acquired between $10000 \mathrm{~cm}^{-1}$ and $380 \mathrm{~cm}^{-1}$ with a $4 \mathrm{~cm}^{-1}$ resolution and averaging over 64 spectra.

\section{Acknowledgments}

We thank Olivier Pluchery for fruitful discussions. We thank Agence Nationale de la Recherche for funding through grant Nanodose and $\mathrm{H} 2 \mathrm{DH}$. This work has been supported by the Region lle-de-France in the framework of DIM Nano-K. This work was supported by French state funds managed by the ANR within 
the Investissements d'Avenir programme under reference ANR-11-IDEX-0004-02, and more specifically within the framework of the Cluster of Excellence MATISSE.

\section{Supplementary information}

The Supporting Information is available free of charge on the ACS Publications website at DOI:

Additional data including material characterization and setup for measurement are given in the supplementary information.

\section{References}

${ }^{1}$ Lhuillier, E. ; Scarafagio, M. ; Hease, P. ; Nadal, B. ; Aubin, H. ; Xu, X. Z. ; Lequeux, N. ; Patriache, G. ; Ithurria, S. ; Dubertret, B. Infrared Photodetection Based on Colloidal Quantum-Dot Films with High Mobility and Optical Absorption up to THz Nano Lett. 2016, 16, 1282-1286.

2 Talapin, D. V.; Lee, J. S.; Kovalenko, M. V.; Shevchenko, E. V. Prospects of Colloidal Nanocrystals for Electronic and Optoelectronic Applications Chem. Rev. 2010, 110, 389-458.

${ }^{3}$ Erwin, S. C.; Zu, L.; Haftel, M. I.; Efros, A.L.; Kennedy, T.A.; Norris, D. J. Doping Semiconductor Nanocrystals Nature 2005, 436, 91-94

${ }^{4}$ Ott, F. D.; Spiegel, L.L.; Norris, D.J.; Erwin, S. C. Microscopic Theory of Cation Exchange in CdSe Nanocrystals Phys. Rev. Lett. 2014, $113,156803$.

5 Schimpf, A. M.; Knowles, K. E.; Carroll, G. M.; Gamelin, D. R. Electronic Doping and Redox Potential Tuning in Colloidal Semiconductor Nanocrystals Acc. Chem. Res. 2015, 48, 1929-1937.

${ }^{6}$ Stavrinadis, A.; Pelli Cresi,J. S.; D'Acapito, F.; Magen, C.; Boscherini, F.; Konstantatos, G. Aliovalent Doping in Colloidal Quantum Dots and its Manifestation on their Optical Properties: Surface Attachment versus Structural Incorporation Chem. Mat. 2016, 28, 5384-5393 .

7 Viswanatha, R.; Brovelli, S.; Pandey, A.; Crooker, S.A.; Klimov, V.I. Copper-Doped Inverted Core/Shell Nanocrystals with "Permanent" Optically Active Holes Nano Lett. 2011, 11, 47534758.

${ }^{8}$ De Trizio, L.; Manna, L. Forging Colloidal Nanostructures via Cation Exchange Reactions, Chem. Rev. 2016, asap

9 Jeong, K.S., Deng, Z., Keuleyan, S., Liu, H, Guyot-Sionnest, P. Air-Stable n-Doped Colloidal HgS Quantum Dots J. Phys. Chem. Lett. 2014, 5, 1139-1143.

10 Shen G.; Guyot-Sionnest, P. HgS and HgS/CdS Colloidal Quantum Dots with Infrared Intraband Transitions and Emergence of a Surface Plasmon J. Phys. Chem. C 2016, 120, 11744-11753

${ }^{11}$ Deng, Z., Jeong, K. S., Guyot-Sionnest, P. Colloidal Quantum dots Intraband Photodetectors ACS Nano 2014, 8, 11707-11714.

12 Wang, C. ; Shim, M. ; Guyot-Sionnest, P. Electrochromic Nanocrystal Quantum Dots, Science 2001, 291, 2390-2392.

${ }^{13}$ Rosencher, E.; Vinter, B. Optoélectronique, 2nd ed. Dunod, Paris, 2002.

${ }^{14}$ Lhuillier, E.; Keuleyan, S.; Rekemeyer, P.; Guyot-Sionnest, P. Thermal Properties of mid Infrared Colloidal Quantum Dot Detectors J. Appl. Phys. 2011, 110, 032110. 
15 Liu, H.; Keuleyan, S.; Guyot-Sionnest, P. n-and p-Type HgTe Quantum Dot Films J. Phys. Chem. C 2011, $116,1344-1349$

16 Lhuillier, E.; Keuleyan, S.; Zolotavin, P.; Guyot-Sionnest, P. Mid-Infrared $\mathrm{HgTe} / \mathrm{As}_{2} \mathrm{~S}_{3} \mathrm{FETs}$ and photodetectors Adv. Mat. 2013, 25, 137.

17 Lhuillier, E.; Keuleyan, S.; Guyot-Sionnest, P. Optical Properties of HgTe Colloidal Quantum Dots Nanotechnology 2012, 23, 175705.

${ }^{18}$ To estimate the magnitude of the 1 s contribution to the total interband signal, we first substract a baseline to the total absorption and then further integrate the area corresponding to the interband $1 \mathrm{~s}$ state. Similarly the contribution of the intraband peak is integrated. We eventually compare the areas of these two peaks in figure $2 c$

${ }^{19}$ Deng, Z. ; Guyot-Sionnest,P. Intraband Luminescence from HgSe/CdS Core/Shell Quantum Dots ACS Nano 2016, 10, 2121-2127

${ }^{20}$ Deboer, B.; Hadipour, A.; Mandoc, M.M.; van Woudenbergh, T.; Blom, P.W. M. Tuning of Metal Work Functions with Self-Assembled Monolayers Adv. Mat. 2005, 17, 621-625

${ }^{21}$ Soreni-Harari, M.; Yaacobi-Gross, N.; Steiner, D.; Aharoni, A.; Banin, U.; Millo, O.; Tessler, N.; Tuning Energetic Levels in Nanocrystal Quantum Dots through Surface Manipulations. Nano Lett. 2008, 8, 678-684

${ }^{22}$ Brown, P.R.; Kim, D.; Lunt, R. R.; Zhao, N.; Bawendi, M. G.; Grossman, J.C.; Bulovic, V. Energy Level Modification in Lead Sulfide Quantum Dot Thin Films through Ligand Exchange, ACS Nano 2014,8, 5863-5872

${ }^{23}$ Miller, E. M.; Kroupa, D. M.; Zhang, J.; Schulz, P.; Marshall, A.R.; Kahn, A.; Lany, S.; Luther, J. M.; Beard, M. C.; Perkins, C. L.; van de Lagemaat, J. Revisiting the Valence and Conduction Band Size Dependence of PbS Quantum Dot Thin Films, ACS Nano 2016, 10, 3302-3311.

${ }^{24}$ Yang, S.; Prendergast, D.; Neaton, J.B. Tuning Semiconductor Band Edge Energies for Solar Photocatalysis via Surface Ligand Passivation Nano Lett. 2012, 12, 383-388.

25 Nethercot, A. H. Jr. Prediction of Fermi Energies and Photoelectric Thresholds Based on Electronegativity Concepts Phys. Rev. Lett. 1974, 33, 1088.

${ }^{26}$ Anderson, N. C.; Hendricks, M. P.; Choi, J. J.; Owen, J. S. Ligand Exchange and the Stoichiometry of Metal Chalcogenide Nanocrystals: Spectroscopic Observation of Facile Metal-Carboxylate Displacement and Binding J. Am. Chem. Soc. 2013, 135, 18536-18548.

27 Arefi, H. H.; Nolan, M.; Fagas, G. Role of the Head and/or Tail Groups of Adsorbed-[Xheadgroup]-Alkyl-[Xtailgroup] $[\mathrm{X}=\mathrm{O}(\mathrm{H}), \mathrm{S}(\mathrm{H}), \mathrm{NH}(2)]$ Chains in Controlling the Work, Function of the Functionalized H:Si(111) Surface J. Phys. Chem. C 2015, 119, 11588-11597.

${ }^{28}$ Arefi, H.H.; Fagas, G. Chemical Trends in the Work Function of Modified Si(111) Surfaces: A DFT Study J. Phys. Chem. C 2014, 118, 14346-14354.

${ }^{29}$ Koh, W.K.; Saudari, S. R.; Fafarman, A. T.; Kagan, C.R.; Murray, C.B. Thiocyanate-Capped PbS Nanocubes: Ambipolar Transport Enables Quantum Dot Based Circuits on a Flexible Substrate Nano Lett. 2011, 11, 4764-4767

${ }^{30}$ Kim, H.; Cho, K.; Kim, D. W.; Lee, H. R.; Kim, S. Bottom- and Top-Gate Field-Effect Thin-Film Transistors with $p$ Channels of Sintered HgTe Nanocrystals Appl. Phys. Lett. 2006, 89, 17, 173107

${ }^{31}$ Kim, H.; Kim, D. W.; Cho, K.; Kim, S. HgTe Nanocrystal-based Thin-Film Transistors Fabricated on Glass Substrates IEEE Electron Device Lett. 2007, 28, 42-44

${ }^{32}$ Jang, J.; Cho, K.; Lee, S. H.; Kim, S. Transparent and Flexible Thin-Film Transistors with Channel Layers Composed of Sintered HgTe Nanocrystals Nanotechnology. 2008, 19, 015204. 
${ }^{33}$ Kim, D. W.; Jang, J.; Kim, H.; Cho, K.; Kim, S. Electrical Characteristics of HgTe Nanocrystal-based Thin Film Transistors Fabricated on Flexible Plastic Substrates Thin Solid Films. 2008, 516, 77157719.

${ }^{34}$ Seong, H.; Cho, K.; Kim, S. Photocurrent Characteristics of Solution-Processed HgTe nanoparticle Thin Films Under the Illumination of $1.3 \mu \mathrm{m}$ Wavelength Light Semicond. Sci. Technol. 2008, 23, 7, 075011 


\section{TOC}
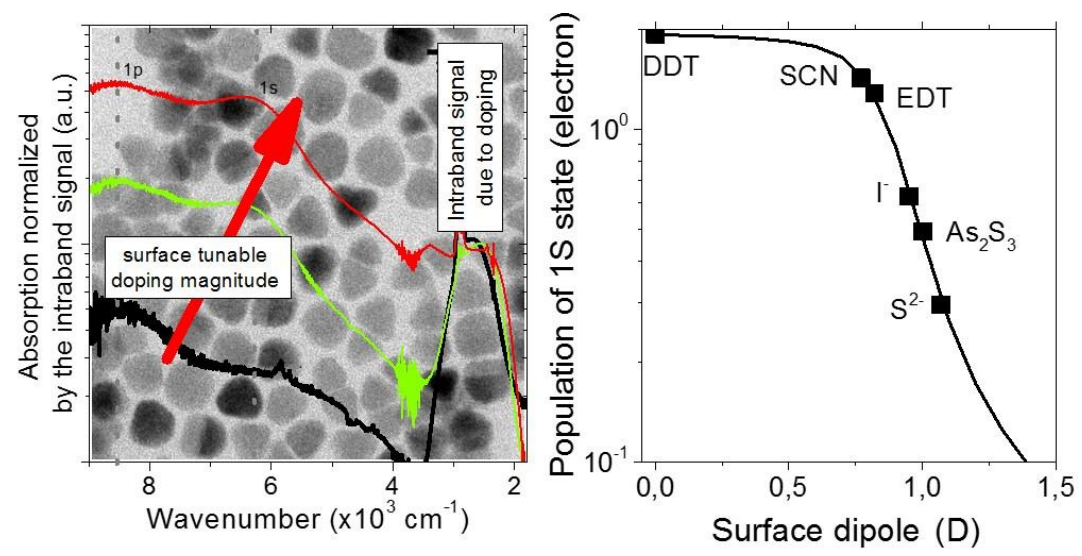